\title{
COMPLICAÇÕES RELACIONADAS AOS TRATAMENTOS DE LESÕES PANCREÁTICAS
}

\section{ARTIGO DE REVISÃO}

DIRANE, Victória Pacheco ${ }^{1}$, RÊGO, Danielly Gonçalves da Silva² ${ }^{2}$ TEODORO, Flávia Pavani $^{3}$, MOYSES, Izabela Cristiane Toledo Diniz ${ }^{4}$, MESTRINER, Luigi Ribeiro ${ }^{5}$, FERREIRA, Raíssa Brum ${ }^{6}$, RODRIGUES, Raizza Montanari ${ }^{7}$, NUNES, Thaynan

${ }^{1}$ Discente do curso de Medicina da Universidade Municipal de São Caetano do Sul, campus Centro, São Caetano do Sul - São Paulo.

2 Discente do curso de Medicina da Universidade Federal do Oeste da Bahia, campus Reitor Edgar Santos, Barreiras - Bahia.

${ }^{3}$ Discente do curso de Medicina da Universidade José do Rosário Vellano, campus Alfenas, Alfenas - Minas Gerais.

${ }^{4}$ Discente do curso de Medicina da Escola Superior de Ciências da Santa Casa de Misericórdia de Vitória, campus Vitória, Vitória - Espírito Santo.

${ }^{5}$ Discente do curso de Medicina da Universidade Municipal de São Caetano do Sul, campus Centro, São Caetano do Sul - São Paulo.

${ }^{6}$ Discente do curso de Medicina da Escola Superior de Ciências da Santa Casa de Misericórdia de Vitória, campus Vitória, Vitória - Espírito Santo.

7 Discente do curso de Medicina do Centro Universitário do Espírito Santo - UNESC, campus Colatina, Colatina - Espírito Santo.

$\mathrm{RC}: 88449$

Disponível em: https://www.nucleodoconhecimento.com.br/saude/lesoes-pancreaticas 
Oliveira ${ }^{8}$, MARCONI, Victor Toniolo ${ }^{9}$, OLIVEIRA, Igor de Sousa ${ }^{10}$, VASCONCELOS, Gilberto Loiola de ${ }^{11}$

DIRANE, Victória Pacheco. Complicações relacionadas aos tratamentos de lesões pancreáticas. Revista Científica Multidisciplinar Núcleo do Conhecimento. Ano 06, Ed. 06, Vol. 07, pp. 127-154. Junho de 2021. ISSN: 2448-0959, Link de acesso: https://www.nucleodoconhecimento.com.br/saude/lesoes-pancreaticas, DOI: 10.32749/nucleodoconhecimento.com.br/saude/lesoes-pancreaticas

\section{RESUMO}

Contexto: O pâncreas é um órgão que pode dar origem a diversas neoplasias, sendo a mais comum o adenocarcinoma ductal, uma neoplasia maligna. Nessa perspectiva, pode-se citar o adenocarcinoma ductal é localizado na maioria das vezes na cabeça do pâncreas, seguida do acometimento de corpo e cauda em outros casos. O único tratamento que possibilita a cura é o cirúrgico, porém, nem todos os pacientes são candidatos a esse tratamento e as complicações intra e pós-operatórias são frequentes. Segundo a Organização Mundial de Saúde (OMS), o câncer de pâncreas é a sétima causa de morte em homens e mulheres em todo o mundo. Apenas 15 a $20 \%$ dos pacientes são candidatos ao tratamento cirúrgico, devido ao diagnóstico tardio da doença. Objetivo: Reconhecer as complicações operatórias relacionadas a lesões císticas do pâncreas. Metodologia: Caracteriza-se como uma revisão

${ }^{8}$ Discente do curso de Medicina da Universidade José do Rosário Vellano, campus Alfenas, Alfenas - Minas Gerais.

${ }^{9}$ Discente do curso de Medicina da Universidade Positivo, campus Ecoville, Curitiba - Paraná.

10 Discente do curso de Medicina da Universidade Federal de Campina Grande, Centro de Formação dos Professores, Cajazeiras - Paraíba.

${ }^{11}$ Orientador. Médico residente pela Universidade Federal do Ceará na Santa Casa de Misericórdia de Sobral.

$\mathrm{RC}: 88449$

Disponível em: https://www.nucleodoconhecimento.com.br/saude/lesoes-pancreaticas 
bibliográfica do tipo integrativa, com abordagem qualitativa e com natureza aplicada. A busca dos artigos foi realizada por meio do acesso à base de dados PUBMED. Para isso, utilizou-se de descritores, contidos no DeCS (Descritores em Ciência da Saúde), referentes aos termos "carcinoma ductal pancreático", "neoplasias pancreáticas", "terapêutica" e "diagnóstico". Tais descritores foram pesquisados nos idiomas português, inglês e espanhol e associados sinônimos pertinentes e operadores booleanos, sendo eles: "parênteses", "AND" e "OR". Principais resultados: Foram analisados 15 estudos e esclareceu-se as principais complicações relacionadas às diferentes escolhas cirúrgicas para o tratamento de câncer de pâncreas como a fístula pancreática, retardo do esvaziamento gástrico, colangite após colocação de stent biliar em obstrução biliar por câncer pancreático ressecável limítrofe assim como as complicações gerais da terapia neoadjuvante em adenocarcinoma pancreático complicações gerais da pancreaticoduodenectomia combinada com ressecção da veia porta (VP) ou da veia mesentérica superior (VMS). Conclusão: Portanto, além de abrir caminhos para novas discussões acerca do tema, pode auxiliar no estabelecimento das condutas cirúrgicas mais adequadas em quadros de câncer de pâncreas ao fazer uma análise comparativa entre a aplicação das técnicas cirúrgicas, reconhecendo as principais complicações operatórias relacionadas a lesões císticas do pâncreas, principalmente quando correlacionadas com o prognóstico do paciente.

Palavras-Chave: Carcinoma Ductal Pancreático, Cirurgia, Complicações, Pâncreas.

\section{INTRODUÇÃO}

O pâncreas é um órgão que pode dar origem a diversas neoplasias, sendo a mais comum o adenocarcinoma ductal, uma neoplasia maligna. Cerca de $85 \%$ a $90 \%$ das neoplasias pancreáticas diagnosticadas são do tipo adenocarcinoma ductal e seus subtipos (LONGNECKER, 2021). O diagnóstico é feito por meio da confirmação histopatológica de uma amostra biopsiada, e seu prognóstico depende do grau histológico do tumor (grau de diferenciação e prevalência de células mitóticas) e da extensão da disseminação dele (estágio do tumor, metástase etc.) É necessário

RC: 88449

Disponível em: https://www.nucleodoconhecimento.com.br/saude/lesoes-pancreaticas 
determinar a área neoplásica e sua localização pela avaliação do tamanho do tumor primário e sua extensão $(T)$, do acometimento de gânglios linfáticos adjacentes $(\mathrm{N})$ e da presença de metástases para áreas distantes (M).

Nessa perspectiva, pode-se citar o adenocarcinoma ductal é localizado na maioria das vezes na cabeça do pâncreas, seguida do acometimento de corpo e cauda em outros casos. O único tratamento que possibilita a cura é o cirúrgico, porém, nem todos os pacientes são candidatos a esse tratamento e as complicações intra e pós-operatórias são frequentes.

Segundo a Organização Mundial de Saúde (OMS), o câncer de pâncreas é a sétima causa de morte em homens e mulheres em todo o mundo, sendo apenas 15 a 20\% dos pacientes são candidatos ao tratamento cirúrgico devido ao diagnóstico tardio da doença. Após surgir o procedimento de Whipple, a mortalidade perioperatória ocorre em menos de $2 \%$ dos casos, porém a morbidade relacionada aos procedimentos continua elevada, ocorrendo entre 30 a $50 \%$ dos casos. As complicações pósoperatórias mais frequentes são retardo do esvaziamento gástrico (18\%), fístula do pâncreas (12\%), infecção da ferida (7\%), abscesso intra-abdominal (6\%), eventos cardíacos (3\%), vazamento biliar (2\%) e reoperação geral (3\%) (TOWNSEND et al., 2015).

Nas complicações mais frequentes, o retardo no esvaziamento gástrico pode ser manifestado por vômitos após a retomada da ingestão de líquidos no pós-operatório. Já na fístula pancreática, o achado que sugere essa complicação é um conteúdo 3 vezes maior de amilase detectada no fluido do dreno pancreático, em relação ao limite superior de amilase sérica total, no terceiro dia após a cirurgia.

As lesões císticas do pâncreas apresentam prognósticos diversificados e constituem um amplo espectro de doenças com características clínicas específicas e prejudiciais ao desenvolvimento homeostático do paciente, bem como após adoção do tratamento cirúrgico do paciente. Diante dessa percepção, levanta-se a seguinte questão: "Qual a relevância de reconhecer as principais complicações intraoperatórias e pósRC: 88449

Disponível em: https://www.nucleodoconhecimento.com.br/saude/lesoes-pancreaticas 
operatórias quando correlacionadas ao estadiamento do paciente com o intuito de instituir um prognóstico mais benéfico?".

Diante desse cenário, é válido ressaltar que a lesão cística do pâncreas tem sido cada vez mais diagnosticada previamente em virtude da evolução tecnológica dos exames de imagem. Dessa forma, tal situação é significativa pelo fato de haver a descoberta na tomografia computadorizada (TC) ou na ressonância magnética (RM), de forma acidental, a identificação de cistos não neoplásicos, cujo tratamento é mais comum em abordagem sintomática ou neoplasia cística quando há a necessidade de ressecamento, retardando, consequentemente, o melhor prognóstico. (FALQUETO et al, 2018). Todavia, deve-se atentar não só aos métodos diagnósticos e de tratamento, mas também às complicações existentes no desenvolvimento de tratamentos cirúrgicos abordados, uma vez que o prognóstico do paciente depende notoriamente do controle dessas complicações.

Desse modo, o objetivo deste estudo consiste em reconhecer as complicações operatórias relacionadas a lesões císticas do pâncreas, principalmente quando correlacionadas com o prognóstico do paciente.

\section{METODOLOGIA}

Este estudo caracteriza-se como uma revisão bibliográfica do tipo integrativa, com abordagem qualitativa, natureza aplicada, procedimento bibliográfico e com objetivo descritivo.

A revisão bibliográfica foi realizada utilizando a base de dados PUBMED e seus sites aliados. Primeiramente, foi feita uma análise dos descritores relevantes para o tema por meio do vocabulário disponível no DeCS (Descritores em Ciência da Saúde), sendo esses: "carcinoma ductal pancreático", "neoplasias pancreáticas", "terapêutica" e "diagnóstico" nos idiomas português, inglês e espanhol. Além dessa seleção, a fim de aperfeiçoar os estudos que melhor contribuíssem para a pesquisa, foi feito uso de

RC: 88449

Disponível em: https://www.nucleodoconhecimento.com.br/saude/lesoes-pancreaticas 
sinônimos pertinentes e operadores booleanos, sendo eles: "parênteses", "AND" e "OR".

Por conseguinte, foram filtrados, em ordem sequencial, os artigos que estavam disponíveis na íntegra, indexados com recorte temporal contido nos últimos cinco anos e nos seguintes idiomas: espanhol, inglês e português. De acordo com os resultados obtidos a partir da fórmula de busca, foram encontrados 49 artigos no PUBMED.

Os critérios de inclusão empregados foram: artigos que elucidassem pacientes, crianças ou adultos, que apresente o manejo do carcinoma ductal pancreático e que aborde as lesões císticas, bem como terapêuticas para tal doença, inclusas no recorte temporal pré-determinado. Ressalta-se que a inclusão desses pacientes independia de etnia ou sexo. Excluíram-se artigos que abordassem somente pacientes com lesões hepáticas e possíveis outras complicações além das lesões císticas. Exclui-se também artigos de opinião, estudos duplicados e artigos que não estavam disponíveis na íntegra.

Diante disso, os pesquisadores, ISO e RMR, avaliaram todos os títulos e resumos dos artigos e selecionaram os estudos baseados nos critérios de elegibilidade citados. Com isso, selecionou-se 15 artigos que contemplavam o objetivo da respectiva pesquisa.

Cabe ressaltar que a pesquisa não apresenta caráter prático, não havendo, portanto, a necessidade de submissão ao Comitê de Ética em Pesquisa (CEP).

Para a apresentação dos resultados desta revisão, os achados foram colocados em um quadro contendo os principais aspectos técnicos dos artigos selecionados, sendo esses: título, ano e local de publicação, autoria e principais resultados.

$\mathrm{RC}: 88449$

Disponível em: https://www.nucleodoconhecimento.com.br/saude/lesoes-pancreaticas 


\section{RESULTADOS}

De acordo com a metodologia, dispõe-se a apresentação dos resultados no seguinte quadro abaixo:

\section{Quadro I - Artigos Incluídos no Estudo}

\begin{tabular}{|c|c|c|c|}
\hline $\begin{array}{l}\text { Numeraçã } \\
\text { o }\end{array}$ & Título e autor & $\begin{array}{l}\text { Ano e } \\
\text { local de } \\
\text { publicaçã } \\
0\end{array}$ & Principais resultados \\
\hline I & $\begin{array}{l}\text { Arterial resection and } \\
\text { reconstruction in } \\
\text { pancreatectomy: surgical } \\
\text { technique and outcomes; } \\
\text { ZHANG, Q. et al. }\end{array}$ & $\begin{array}{l}2019 \\
\text { Estados } \\
\text { Unidos da } \\
\text { América. }\end{array}$ & $\begin{array}{l}\text { Pela anatomia do pâncreas, } \\
\text { muitas neoplasias dele } \\
\text { invadem os tecidos } \\
\text { adjacentes, e os principais } \\
\text { alvos são as artérias } \\
\text { hepática (AH) e a } \\
\text { mesentérica superior (MAS). } \\
\text { Com isso, foram avaliados } \\
21 \text { pacientes com tumores } \\
\text { pancreáticos ou na ampola, } \\
\text { que realizaram } \\
\text { pancreatectomia combinada } \\
\text { com ressecção } \\
\text { reconstrução arterial. } \\
\text { Um grande benefício desta } \\
\text { técnica, é o fato de que com } \\
\text { a ressecção, a quimioterapia } \\
\text { neoadjuvante acarreta a }\end{array}$ \\
\hline
\end{tabular}

RC: 88449

Disponível em: https://www.nucleodoconhecimento.com.br/saude/lesoes-pancreaticas 


\begin{tabular}{|c|c|c|c|}
\hline & & & $\begin{array}{l}\text { resultados melhores do que } \\
\text { sem a ressecção. } \\
\text { Por isso, foi relatado que a } \\
\text { pancreatectomia combinada } \\
\text { com a ressecção e } \\
\text { reconstrução arterial seria } \\
\text { um meio de tratamento que } \\
\text { poderia ser considerado aos } \\
\text { pacientes com } \\
\text { determinada patologia. }\end{array}$ \\
\hline II & $\begin{array}{l}\text { Comparison of } \\
\text { Laparoscopic and Open } \\
\text { Pancreaticoduodenecto } \\
\text { my for the Treatment of } \\
\text { Nonpancreatic } \\
\text { Periampullary } \\
\text { Adenocarcinomas; } \\
\text { MENG, L. W. }\end{array}$ & $\begin{array}{l}2018, \\
\text { Estados } \\
\text { Unidos da } \\
\text { América. }\end{array}$ & $\begin{array}{l}\text { A única cura de } \\
\text { adenocarcinomas } \\
\text { periampulares pancreáticos } \\
\text { é } \\
\text { pancreaticoduodenectomia } \\
\text { (PD), e esta cirurgia existe } \\
\text { tanto por laparoscopia (LPD) } \\
\text { quanto aberta (OPD). } \\
\text { A amostra do estudo foram } \\
58 \text { pacientes que fizeram a } \\
\text { LPD e } 58 \text { que fizeram a OPD, } \\
\text { levando em conta que todos } \\
\text { tiveram uma média de } 34 \\
\text { meses de acompanhamento. } \\
\text { Foi concluído que, a LPD e a } \\
\text { OPD têm resultados } \\
\text { parecidos, entretanto a LPD } \\
\text { tem maior morbidade, sendo }\end{array}$ \\
\hline
\end{tabular}




\begin{tabular}{|c|c|c|c|}
\hline & & & 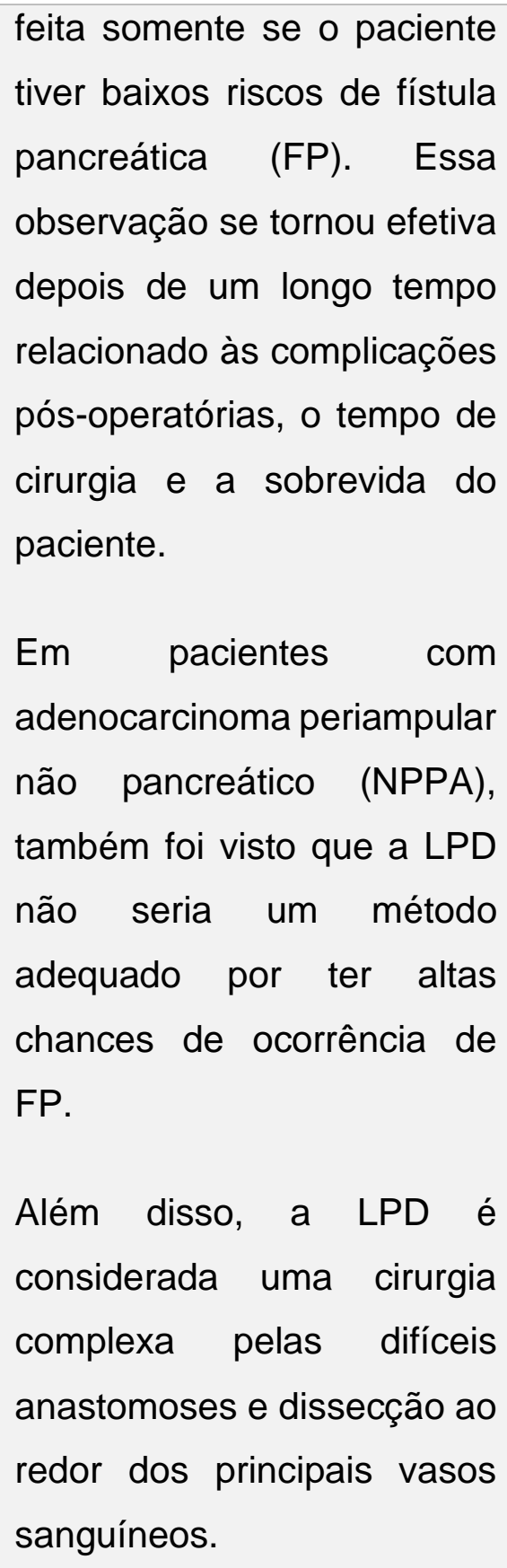 \\
\hline III & $\begin{array}{l}\text { Expanding laparoscopic } \\
\text { pancreaticoduodenectom } \\
\text { y to pancreatichead and } \\
\text { periampullary } \\
\text { malignancy: major } \\
\text { findings based on }\end{array}$ & $\begin{array}{l}2018 \\
\text { Inglaterra. }\end{array}$ & $\begin{array}{l}\text { Nesse estudo foram } \\
\text { comparados resultados de } \\
\text { sobrevida a longo prazo, } \\
\text { da pancreaticoduodenecto } \\
\text { mia laparoscópica (LDP) e } \\
\text { da }\end{array}$ \\
\hline
\end{tabular}

RC: 88449

Disponível em: https://www.nucleodoconhecimento.com.br/saude/lesoes-pancreaticas 


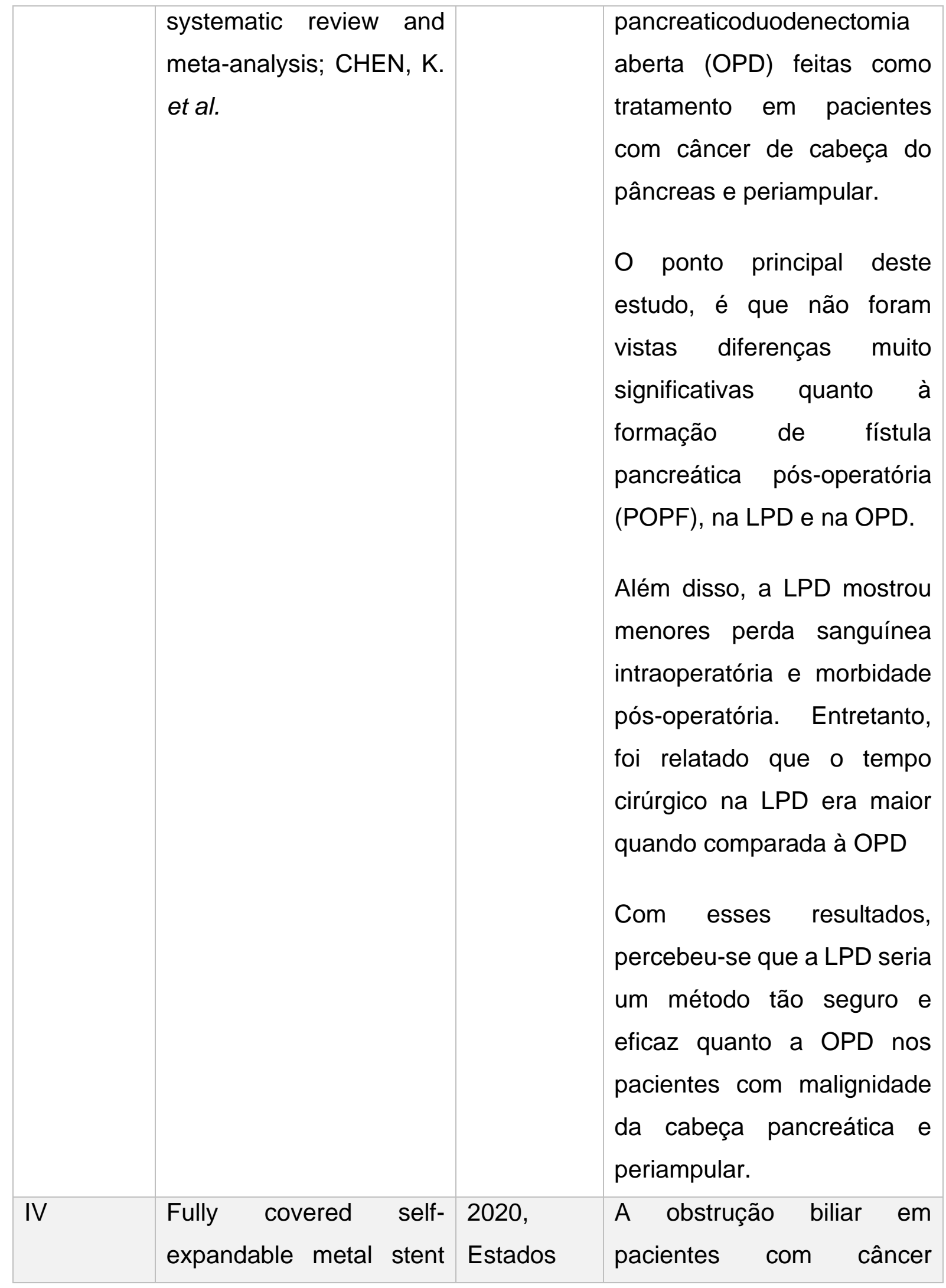

RC: 88449

Disponível em: https://www.nucleodoconhecimento.com.br/saude/lesoes-pancreaticas 


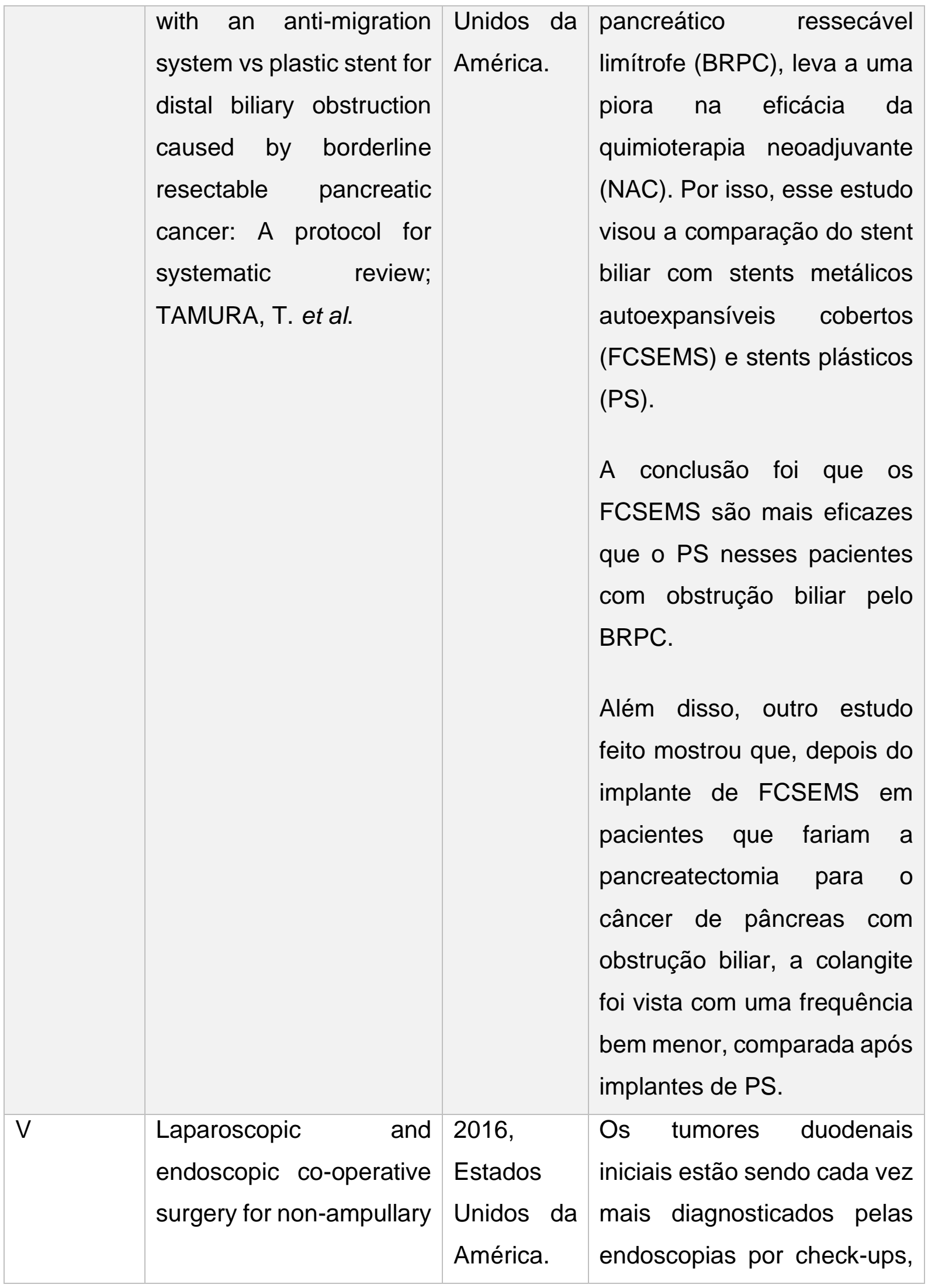

RC: 88449

Disponível em: https://www.nucleodoconhecimento.com.br/saude/lesoes-pancreaticas 


\begin{tabular}{|c|c|}
\hline $\begin{array}{l}\text { duodenal tumors; } \\
\text { ICHIKAWA, D. et al. }\end{array}$ & $\begin{array}{l}\text { por isso, vem sendo } \\
\text { estudado uma maneira } \\
\text { menos invasiva para o } \\
\text { tratamento dos mesmos. } \\
\text { Opção de tratamento menos } \\
\text { invasivas seria a ressecção } \\
\text { endoscópica de tumor ou a } \\
\text { ressecção laparoscópica } \\
\text { local. Dentre os tratamentos } \\
\text { endoscópicos, a } \\
\text { endoscópica da submucosa } \\
\text { (ESD) mostrou ser mais } \\
\text { vantajosa que a endoscópica } \\
\text { da mucosa (EMR). } \\
\text { Entretanto, a ESD é vista } \\
\text { como um método mais difícil } \\
\text { em tumores duodenais, por } \\
\text { aumentar o risco de } \\
\text { sangramento e perfuração } \\
\text { em tumores duodenais } \\
\text { superficiais. } \\
\text { Uma possível perfuração } \\
\text { duodenal acarreta a efusão } \\
\text { de fluidos duodenais na } \\
\text { cavidade abdominal, tendo } \\
\text { como consequência principal } \\
\text { a peritonite, levando ao }\end{array}$ \\
\hline
\end{tabular}

RC: 88449

Disponível em: https://www.nucleodoconhecimento.com.br/saude/lesoes-pancreaticas 


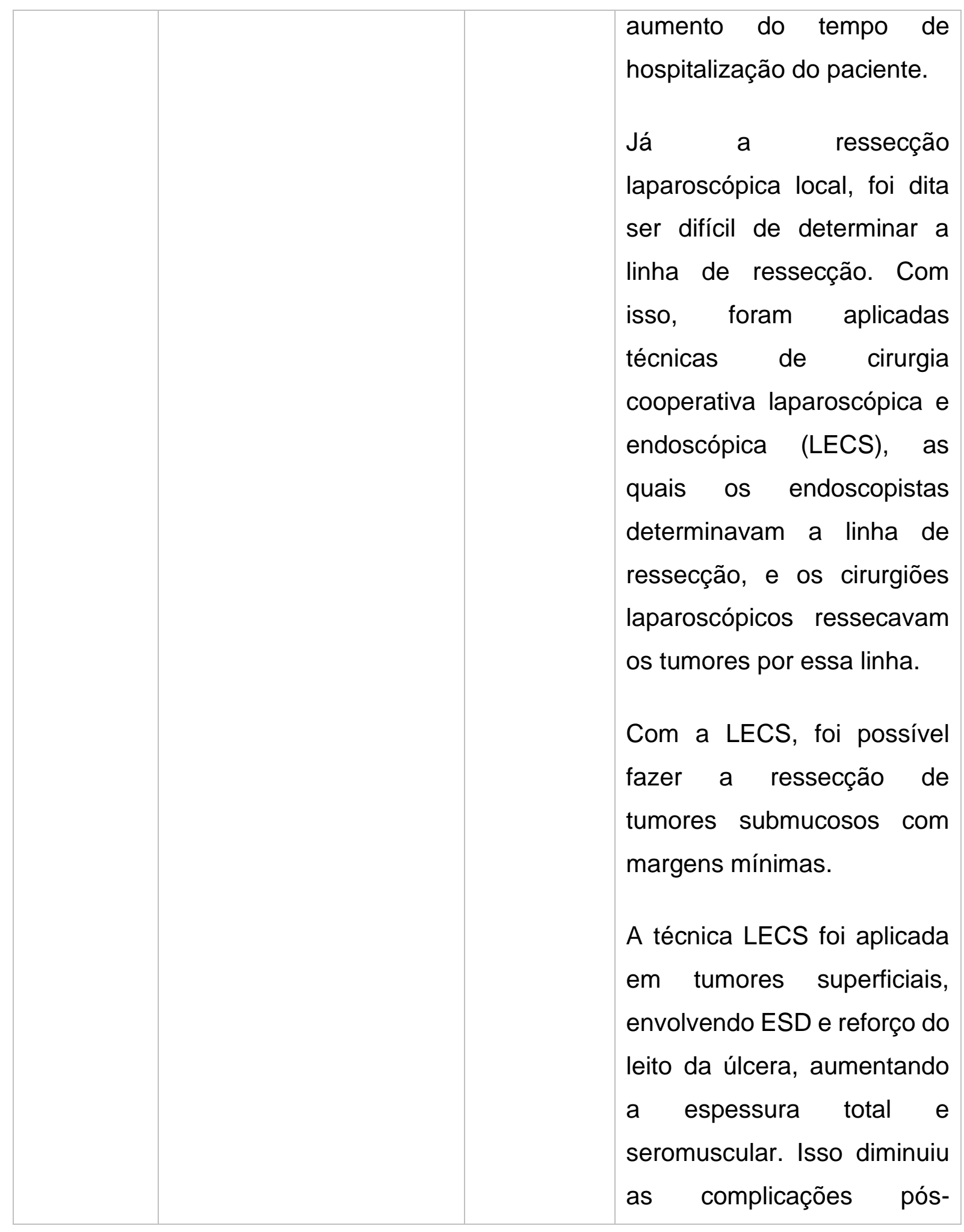

RC: 88449

Disponível em: https://www.nucleodoconhecimento.com.br/saude/lesoes-pancreaticas 


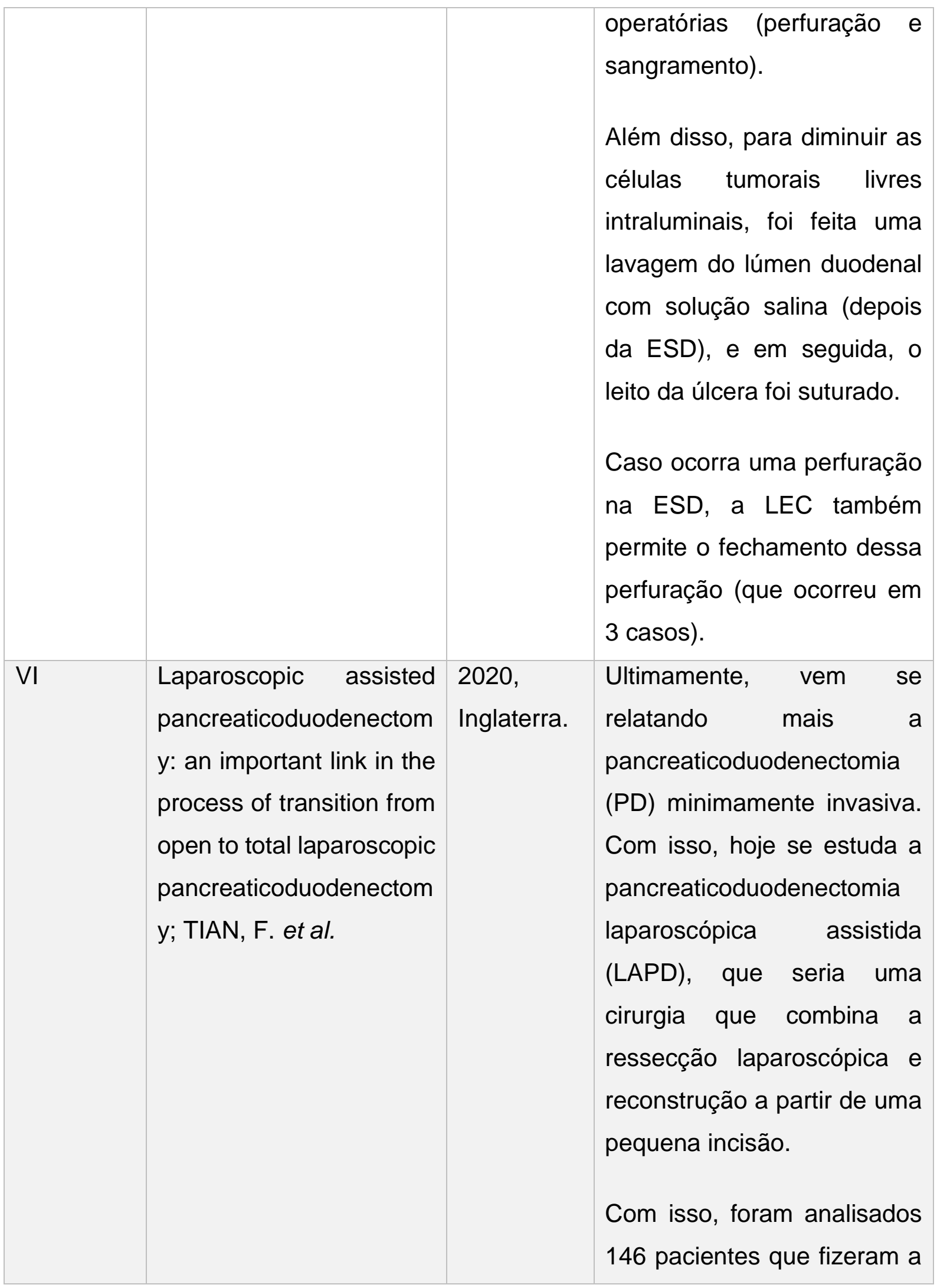

RC: 88449

Disponível em: https://www.nucleodoconhecimento.com.br/saude/lesoes-pancreaticas 


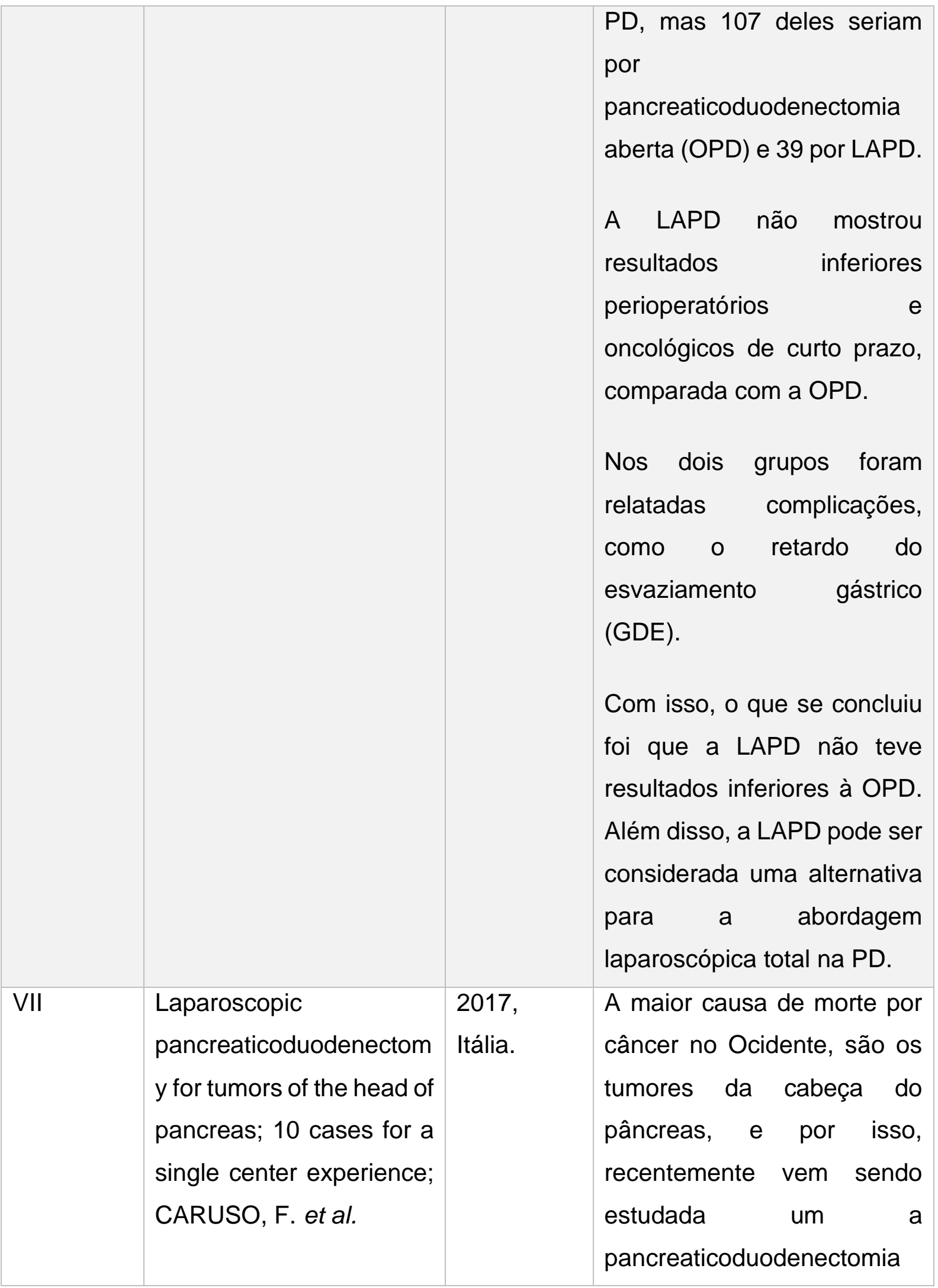

RC: 88449

Disponível em: https://www.nucleodoconhecimento.com.br/saude/lesoes-pancreaticas 


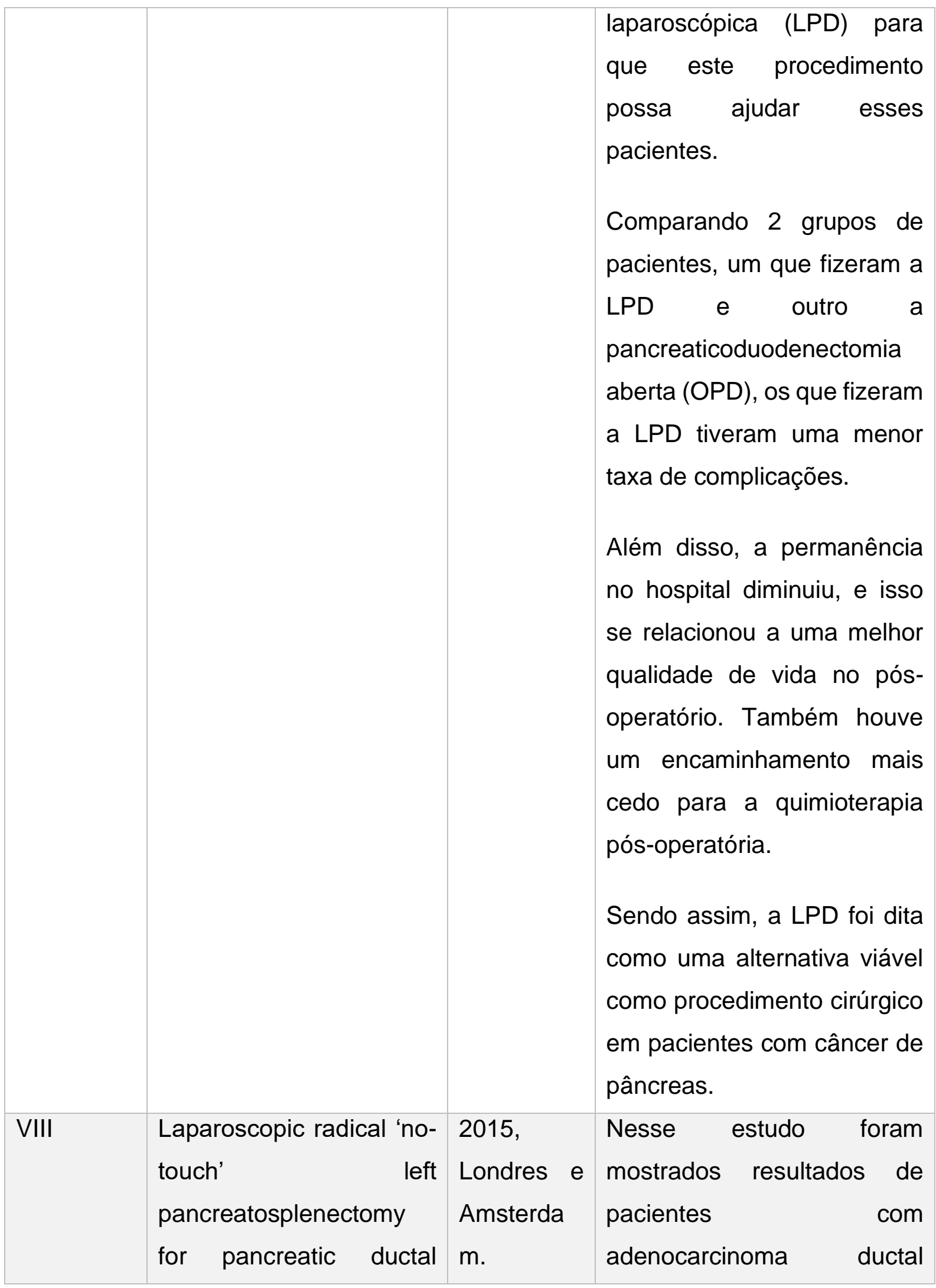

RC: 88449

Disponível em: https://www.nucleodoconhecimento.com.br/saude/lesoes-pancreaticas 


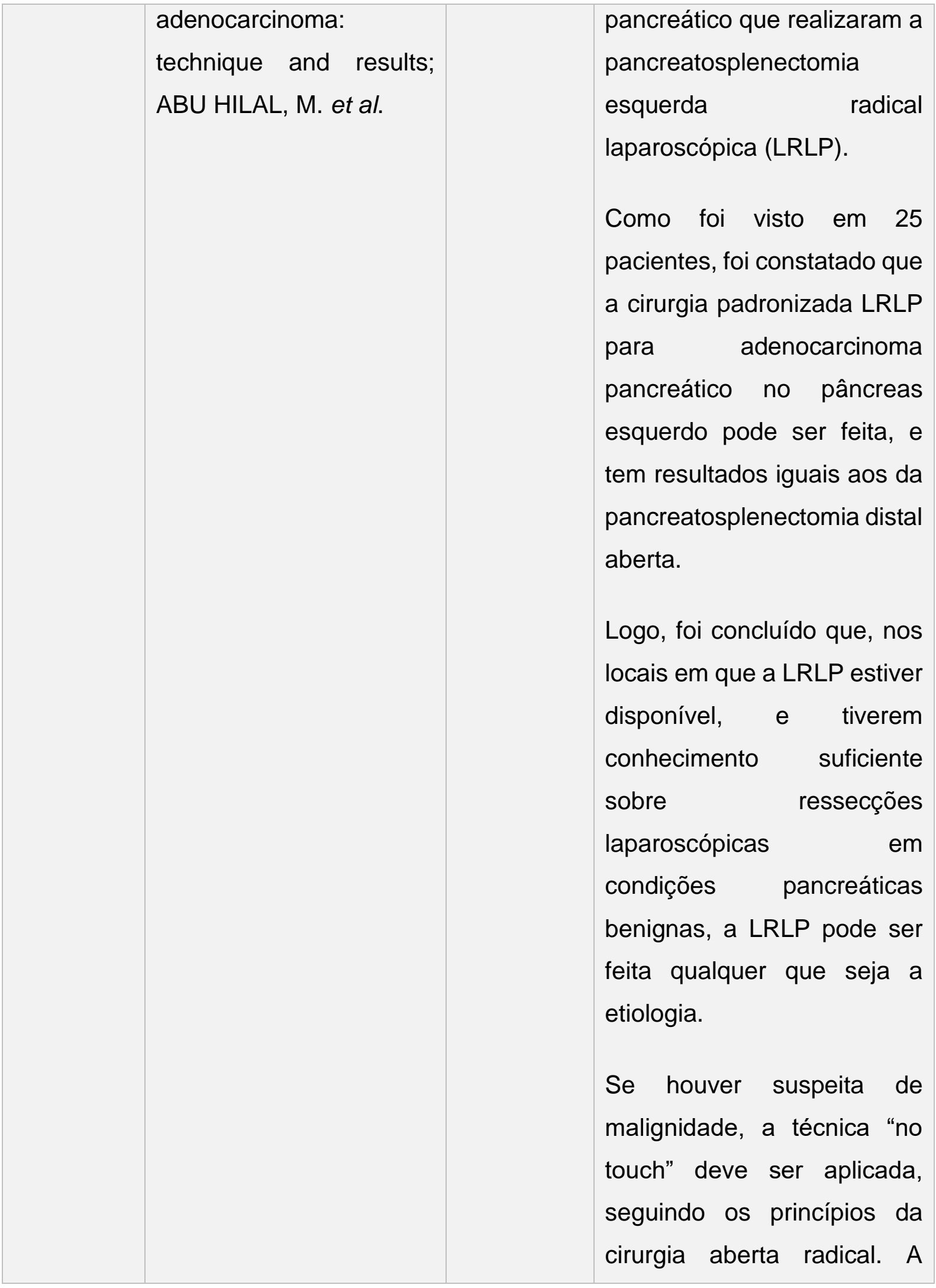

RC: 88449

Disponível em: https://www.nucleodoconhecimento.com.br/saude/lesoes-pancreaticas 


\begin{tabular}{|c|c|c|c|}
\hline & & & $\begin{array}{l}\text { técnica "no touch" previne a } \\
\text { disseminação de células } \\
\text { tumorais, por isso deve ser } \\
\text { realizada nesses casos. }\end{array}$ \\
\hline IX & $\begin{array}{lr}\text { Laparoscopic } & \text { versus } \\
\text { open } & \text { distal } \\
\text { pancreatectomy } & \text { for } \\
\text { pancreatic } & \text { ductal } \\
\text { adenocarcinoma: } & \text { a } \\
\text { single-center experience; } \\
\text { ZHANG, Ab. et al. }\end{array}$ & $\begin{array}{l}2017, \\
\text { China. }\end{array}$ & $\begin{array}{l}\text { Hoje, a ressecção cirúrgica é } \\
\text { o único tratamento curativo } \\
\text { do adenocarcinoma } \\
\text { pancreático, o } \\
\text { procedimento padrão feito é } \\
\text { a pancreatectomia distal. } \\
\text { A pancreatectomia distal } \\
\text { laparoscópica (LDP) é um } \\
\text { procedimento novo que vem } \\
\text { demonstrando segurança. } \\
\text { A LDP foi associada a uma } \\
\text { menor morbidade pós- } \\
\text { operatória, e a um menor } \\
\text { tempo de internação do } \\
\text { paciente, comparada à } \\
\text { técnica convencional. } \\
\text { tão segura e eficaz quanto a } \\
\text { pancreatectomia distal, em } \\
\text { pacientes chegou-se à } \\
\text { adenocarcinoma } \\
\text { pancreático. }\end{array}$ \\
\hline
\end{tabular}

RC: 88449

Disponível em: https://www.nucleodoconhecimento.com.br/saude/lesoes-pancreaticas 


\begin{tabular}{|c|c|c|c|}
\hline & & & $\begin{array}{l}\text { Além disso, a LDP utiliza } \\
\text { uma técnica com } \\
\text { grampeador endo-GIA. Na } \\
\text { pancreatectomia distal } \\
\text { ocorre a secção do pâncreas } \\
\text { através de um bisturi ou } \\
\text { lâmina de eletrocautério. Foi } \\
\text { visto que o fato do uso do } \\
\text { grampeador endo-GIA na } \\
\text { LDP diminuiu a incidência de } \\
\text { fístulas pancreáticas. }\end{array}$ \\
\hline$X$ & $\begin{array}{l}\text { Pancreatic } \\
\text { adenocarcinoma: effects } \\
\text { of neoadjuvant therapy } \\
\text { on post-pancreatectomy } \\
\text { outcomes - an American } \\
\text { College of Surgeons } \\
\text { National Surgical Quality } \\
\text { Improvement Program } \\
\text { targeted variable review; } \\
\text { CZOSNYKA, N. M.; } \\
\text { BORGERT A. J.; SMITH, } \\
\text { T. J. }\end{array}$ & $\begin{array}{l}2017, \\
\text { Inglaterra. }\end{array}$ & $\begin{array}{l}\text { De acordo com as diretrizes } \\
\text { da National Comprehensive } \\
\text { Cancer Network (NCCN), a } \\
\text { cirurgia é a primeira terapia } \\
\text { feita em pacientes com } \\
\text { doença ressecável. Já a } \\
\text { terapia neoadjuvante é } \\
\text { recomendada só em } \\
\text { pacientes com doença } \\
\text { ressecável limítrofe. } \\
\text { Utilizando dados da ACS- } \\
\text { NSQIP (banco de dados que } \\
\text { coleta variáveis } \\
\text { demográficas, pré- } \\
\text { operatórias de } \\
\text { procedimento, com o objetivo } \\
\text { de fornecer taxas de risco de } \\
\text { morbimortalidade }\end{array}$ \\
\hline
\end{tabular}




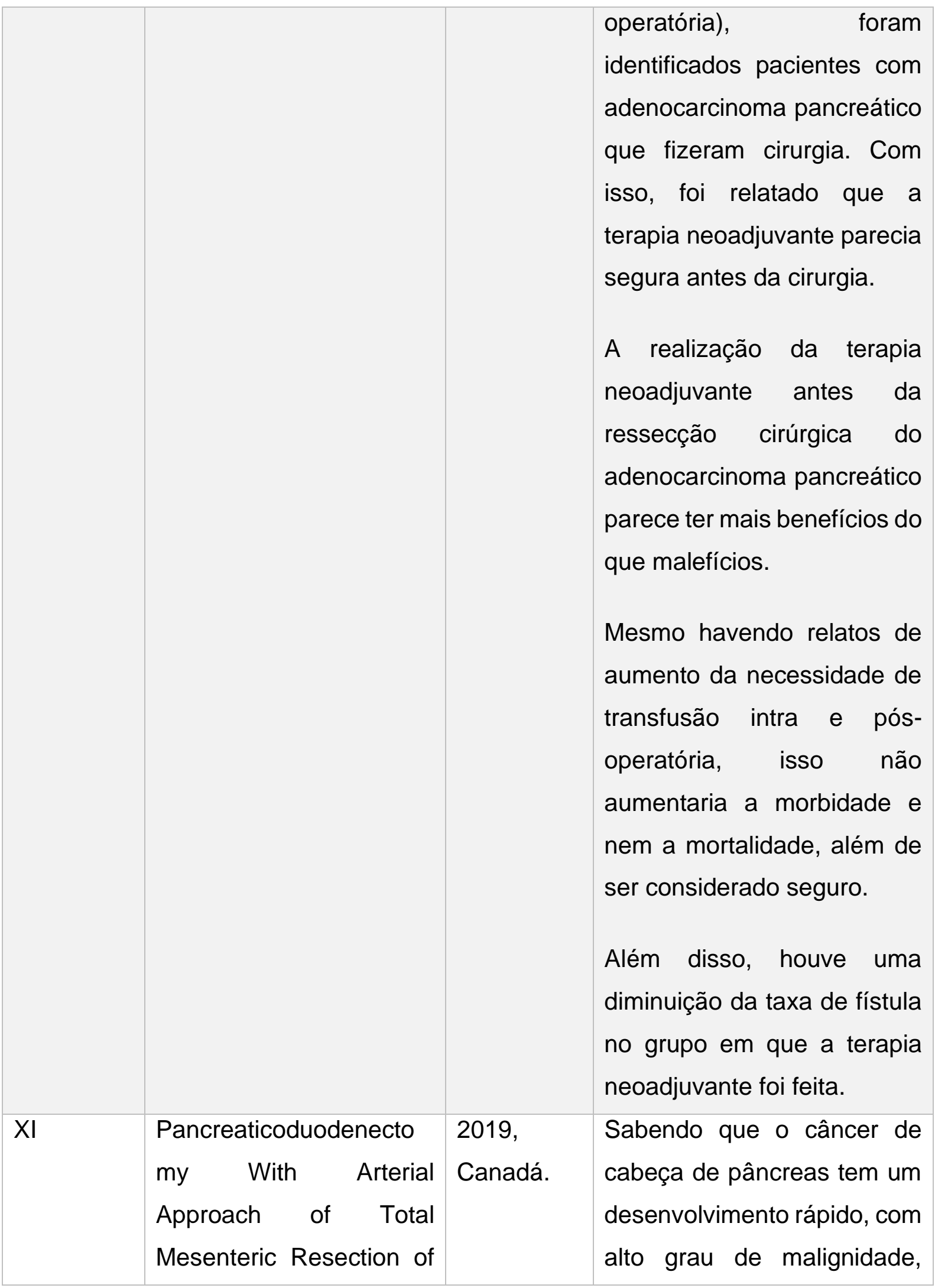

RC: 88449

Disponível em: https://www.nucleodoconhecimento.com.br/saude/lesoes-pancreaticas 


\begin{tabular}{|c|c|}
\hline $\begin{array}{l}\text { the Pancreas for } \\
\text { Pancreatic Head Cancer; } \\
\text { DU, F. et al. }\end{array}$ & $\begin{array}{l}\text { tem sido discutido um outro } \\
\text { procedimento, além da } \\
\text { pancreaticoduodenectomia } \\
\text { (PD) para ajudar os } \\
\text { pacientes com essa } \\
\text { patologia. } \\
\text { Esse estudo analisou } 60 \\
\text { pacientes, os quais } 28 \text { foram } \\
\text { tratados com PD com } \\
\text { excisão total do } \\
\text { mesopâncreas (TMpE), e } 32 \\
\text { só com PD. } \\
\text { Foi relatado que a PD com } \\
\text { TMpE poderia reduzir o } \\
\text { sangramento na cirurgia, e } \\
\text { diminuir o tempo da mesma. } \\
\text { Além disso, esse } \\
\text { procedimento faz com que } \\
\text { seja mais fácil a visualização } \\
\text { da parte posterolateral do } \\
\text { tumor na cabeça do } \\
\text { pâncreas, e isso diminuiu a } \\
\text { tração do tumor. } \\
\text { Também foi visto que, em } \\
\text { pacientes com suspeita do } \\
\text { acometimento da veia porta } \\
\text { (VP) e da veia mesentérica } \\
\text { superior (VMS), a PD com }\end{array}$ \\
\hline
\end{tabular}

RC: 88449 


\begin{tabular}{|c|c|c|c|}
\hline & & & $\begin{array}{l}\text { TMpE evitou a disseminação } \\
\text { das células tumorais através } \\
\text { da veia porta. } \\
\text { Portando, foi concluído que a } \\
\text { PD com TMpE por via } \\
\text { arterial, pode ser feita como } \\
\text { tratamento padrão para } \\
\text { câncer de pâncreas por } \\
\text { diversas vantagens como, } \\
\text { diminuição de lesões } \\
\text { cirúrgicas, do sangramento } \\
\text { cirúrgico e do tempo de } \\
\text { cirurgia. }\end{array}$ \\
\hline XII & $\begin{array}{l}\text { Portal vein/superior } \\
\text { mesenteric vein resection } \\
\text { in pancreatic cancer } \\
\text { treatment in the elderly; } \\
\text { FANG, Jz. et al. }\end{array}$ & $\begin{array}{l}2017, \\
\text { Estados } \\
\text { Unidos da } \\
\text { América. }\end{array}$ & $\begin{array}{l}\text { Pelo câncer pancreático ter } \\
\text { um prognóstico muito } \\
\text { desfavorável, médicos } \\
\text { tentam estender os critérios } \\
\text { cirúrgicos dessa doença, } \\
\text { através de } \\
\text { pancreaticoduodenectomia } \\
\text { (PD) combinada com } \\
\text { ressecção da veia porta (VP) } \\
\text { ou da veia mesentérica } \\
\text { superior (VMS), } \\
\text { reconstrução em pacientes } \\
\text { ressecáveis limítrofes. } \\
\text { Nesse estudo, } 83 \text { pacientes } \\
\text { com } 65 \text { anos ou mais, } \\
\text { fizeram a PD, e foram }\end{array}$ \\
\hline
\end{tabular}

RC: 88449 


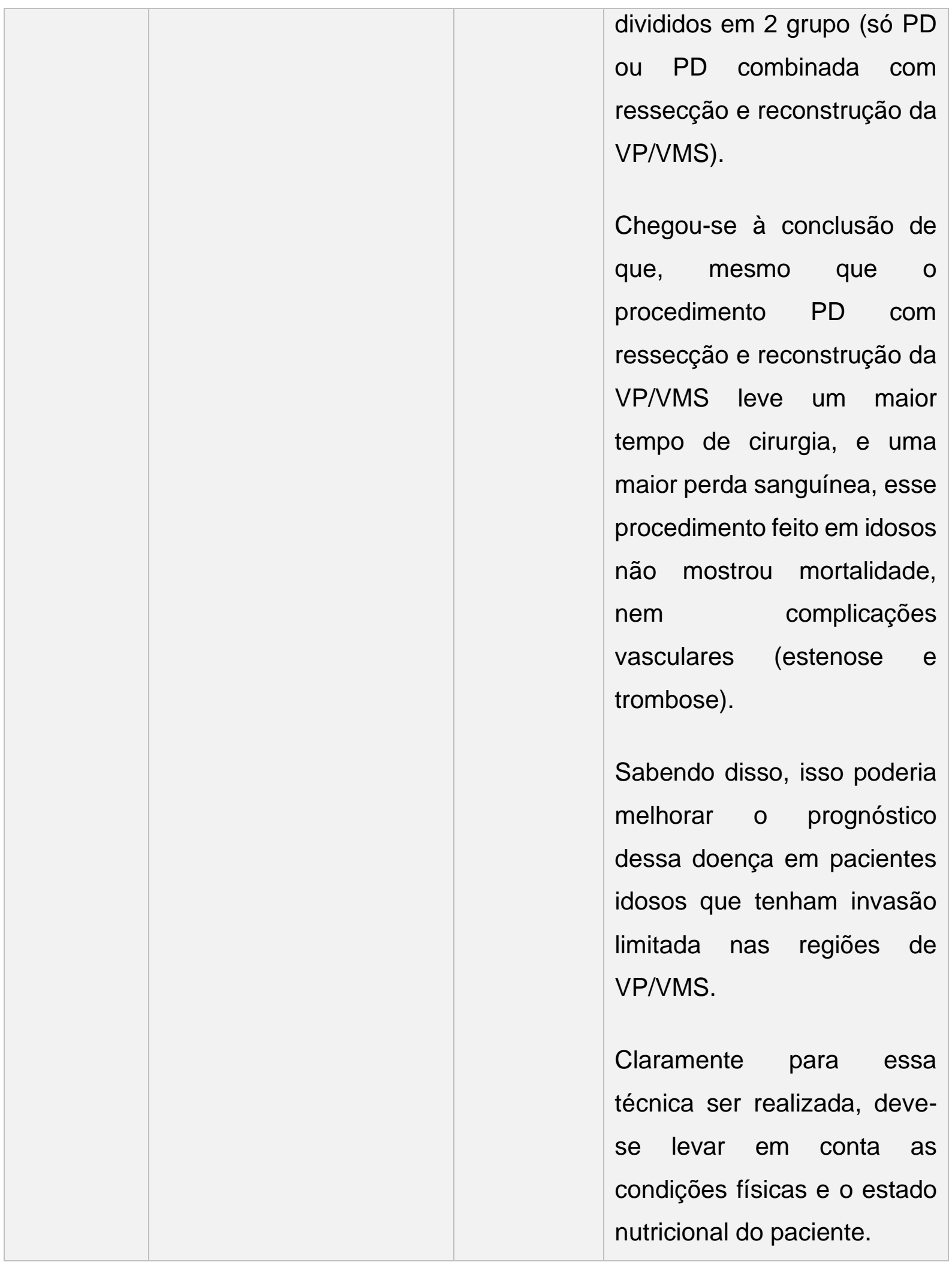

RC: 88449

Disponível em: https://www.nucleodoconhecimento.com.br/saude/lesoes-pancreaticas 


\begin{tabular}{|c|c|c|c|}
\hline XIII & $\begin{array}{l}\text { Protocol of a prospective, } \\
\text { monocentric phase l/II } \\
\text { feasibility study } \\
\text { investigating the safety of } \\
\text { multimodality treatment } \\
\text { with a combination of } \\
\text { intraoperative } \\
\text { chemotherapy and } \\
\text { surgical resection in } \\
\text { locally confined or } \\
\text { borderline resectable } \\
\text { pancreatic cancer: the } \\
\text { combiCaRe study; } \\
\text { ROTH, S. et al. }\end{array}$ & $\begin{array}{l}2019, \\
\text { Reino } \\
\text { Unido. }\end{array}$ & $\begin{array}{l}\text { adenocarcinoma } \\
\text { pancreático é uma patologia } \\
\text { com prognóstico muito ruim. } \\
\text { O tratamento padrão dessa } \\
\text { doença se dá pela ressecção } \\
\text { completa do tumor seguida } \\
\text { de quimioterapia } \\
\text { neoadjuvante, entretanto foi } \\
\text { relatado que a disseminação } \\
\text { das células tumorais pela } \\
\text { manipulação feita cirurgia, } \\
\text { poderia aumentar a taxa de } \\
\text { metástases futuras. } \\
\text { Por isso esse estudo visava } \\
\text { avaliar a segurança de uma } \\
\text { quimioterapia neoadjuvante } \\
\text { em pacientes com câncer } \\
\text { pancreático, em conjunto } \\
\text { com a ressecção do câncer. } \\
\text { O resultado deste estudo não } \\
\text { foi apresentado pelo artigo. }\end{array}$ \\
\hline XIV & $\begin{array}{l}\text { The extent of vascular } \\
\text { resection is associated } \\
\text { with perioperative } \\
\text { outcome in patients } \\
\text { undergoing } \\
\text { pancreaticoduodenectom } \\
\text { y; KANTOR, O. et al. }\end{array}$ & $\begin{array}{l}2018, \\
\text { Inglaterra. }\end{array}$ & $\begin{array}{l}\text { A pancreaticoduodenectomia } \\
\text { (PD) é o único tratamento } \\
\text { curativo disponível para } \\
\text { pacientes com } \\
\text { adenocarcinoma ductal da } \\
\text { cabeça do pâncreas. Com o } \\
\text { avanço da tecnologia, a }\end{array}$ \\
\hline
\end{tabular}




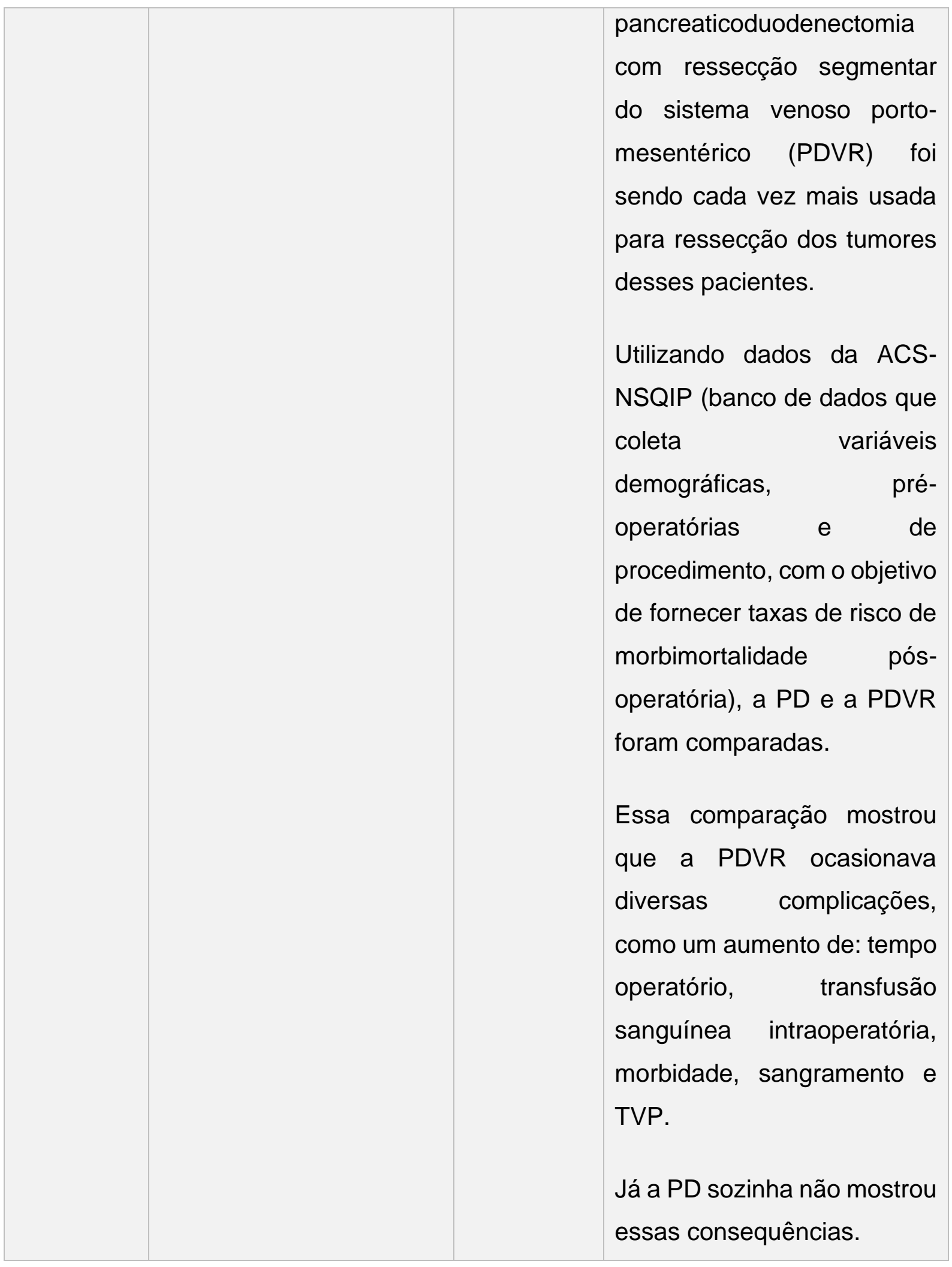

RC: 88449

Disponível em: https://www.nucleodoconhecimento.com.br/saude/lesoes-pancreaticas 


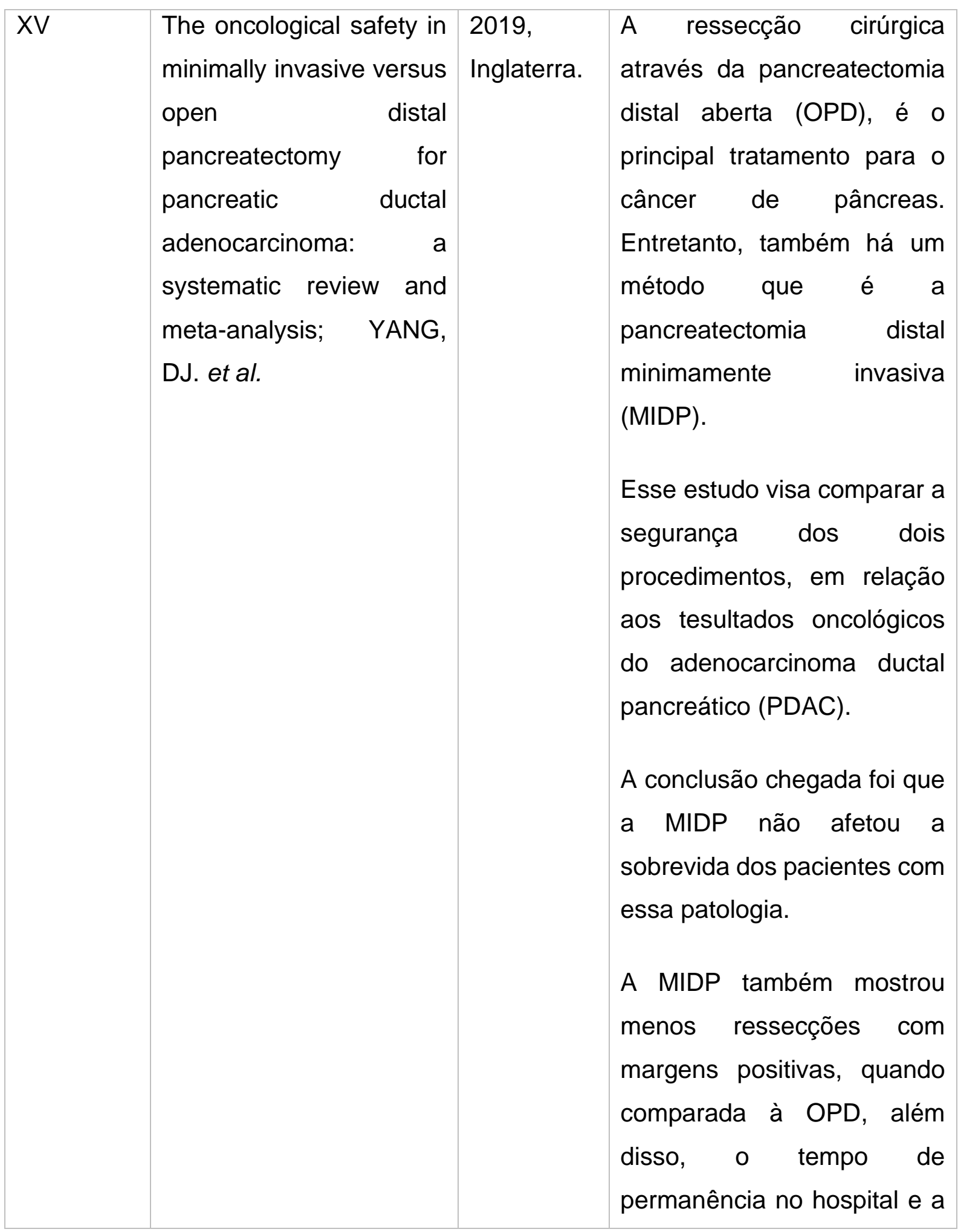

RC: 88449

Disponível em: https://www.nucleodoconhecimento.com.br/saude/lesoes-pancreaticas 


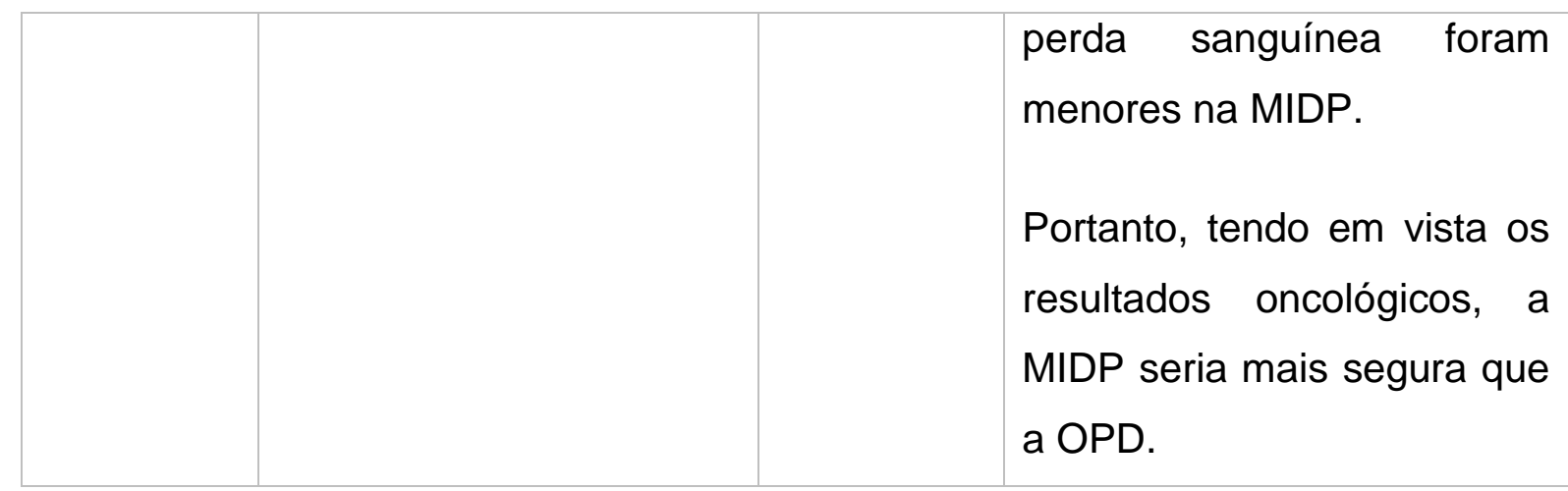

Fonte: Autoria Própria, 2021.

\section{DISCUSSÃO}

\section{CONDUTAS TERAPÊUTICAS}

Existem dois caminhos a serem seguidos em caso de diagnóstico de neoplasia pancreática: a vigilância por exames de imagem e a ressecção cirúrgica. Segundo os critérios de Fukuoka, a vigilância deve ser feita em pacientes com neoplasias pancreáticas que não apresentem estigma de alto-risco para malignidade nem características preocupantes ou resultados negativos e/ou inconclusivos na ultrassonografia endoscópica.

A partir de então, avalia-se o tamanho do cisto para a escolha ideal da sequência de vigilância e o tipo de exame de imagem: cistos $<1 \mathrm{~cm}$ recomenda-se vigilância com tomografia computadorizada (TC) ou ressonância magnética nuclear (RMN) nos primeiros 6 meses pós diagnóstico e, depois, repetir a cada 2 anos sem mudança. Cistos entre 1-2 cm, realizar TC/RMN nos primeiros 6 meses, 1 ano e 2 anos e repetir a cada 2 anos se não houver mudança nas características do cisto. Já para os cistos entre 2-3 cm realizar ultrassonografia endoscópica (USE) nos 3-6 primeiros meses e, depois, a cada 1 ano alternar USE com RMN sendo que nesses casos é recomendado considerar cirurgia para os mais jovens e se atentar com uma vigilância mais prolongada nos pacientes que a necessitam. Por fim, cistos maiores que $3 \mathrm{~cm}$ é indicado USE alternado com RMN a cada 3-6 meses sendo as indicações anteriores

RC: 88449

Disponível em: https://www.nucleodoconhecimento.com.br/saude/lesoes-pancreaticas 
válidas para estes casos. Se indicativo de cirurgia como, por exemplo, características altamente malignas, pode-se escolher entre os métodos de pancreatectomia invasiva ou não invasiva. (TANAKA et al., 2017)

Um outro tipo de conduta terapêutica citada neste estudo de Tanaka et al. (2017), foi a ablação da mucosa por etanol guiada por USE em pacientes com lesão cística maiores que $2 \mathrm{~cm}$ e que não se comuniquem com o ducto principal pancreático ou em pacientes de alto-risco ou que se recusaram realizar a conduta cirúrgica. Entretanto, ainda é necessário maiores estudos relacionados a esse método principalmente devido às complicações pós administração do etanol ou etanol seguido de Paclitaxel para a ablação da mucosa. (TANAKA et al., 2017)

Já no compilado da World Gastroenterology Organization Global Guidelines (2019), diversos aspectos se convergiram para as condutas terapêuticas de neoplasias pancreáticas como a avaliação custo-benefício entre os riscos pós-operatórios no caso da escolha de ressecção e os riscos das malignidades da neoplasia. Os critérios utilizados foram de Sendai - outra forma de guiar as condutas além do critério Fukuoka, mas que apresenta similaridades - cuja indicação para ressecção cirurgia inclui o alto risco de malignidade, os sintomas clínicos, citologia positiva, tamanho do cisto, idade e comorbidades do paciente. Assim como no critério de Fukuoka, a vigilância é necessária e a continuação deve ser feita em longo prazo para todos os tipos de neoplasias. Os métodos para isso são os mesmos encontrados no outro estudo que são tomografia computadorizada, ressonância magnética nuclear, ultrassonografia endoscópica e, em acréscimo, colangiopancreatografia por ressonância magnética. (MALAGELADA et al., 2019)

Por meio da revisão de literatura escolhida para a confecção deste artigo, percebe-se uma convergência geral quanto à conduta terapêutica do tipo cirúrgica para as neoplasias pancreáticas. As principais escolhas incluíam as cirurgias não-invasivas, a exemplo da pancreatosplenectomia esquerda radical laparoscópica (ABU HILAL et al., 2015), pancreatectomia distal minimamente invasiva (YANG et al., 2019), 
pancreaticoduodenectomia laparoscópica (MENG et al., 2018) (CARUSO et al., 2017), sendo, também, comparadas com a conduta por cirurgias abertas. Tais condutas, por sua vez, são realizadas a partir da avaliação das características e extensão da neoplasia, bem como as condições do paciente, a exemplo ressecção cirúrgica por meio da pancreatectomia distal aberta (YANG et al., 2019), pancreaticoduodenectomia aberta (MENG et al., 2018) (CARUSO et al., 2017) (KANTOR et al., 2018).

\section{COMPLICAÇÕES}

\section{FÍSTULA PANCREÁTICA APÓS PANCREATICODUODENECTOMIA}

A fístula pancreática está dentre as principais complicações da pancreaticoduodenectomia, estando relacionada ao aumento da morbidade quando não tratadas ou não tratadas de maneira adequada. O estudo apresentado por Chen et al. (2018) compara a realização da pancreaticoduodenectomia aberta (OPD) e laparoscopicamente (LPD) quanto a formação de fístula pancreática no pósoperatório, evidenciando que não houve diferenças significativas. No entanto, Meng (2018) observou que as LPD apresentaram maior propensão à fístula pancreática, sendo a complicação justificada pelas complexas anastomoses e dissecções ao redor dos principais vasos sanguíneos.

\section{COLANGITE APÓS COLOCAÇÃO DE STENT BILIAR EM OBSTRUÇÃO BILIAR POR CÂNCER PANCREÁTICO RESSECÁVEL LIMÍTROFE}

A revisão sistemática de Tamura et al. (2020) discorre acerca do uso de stents em pacientes portadores de obstrução biliar por câncer pancreático limítrofe (BRPC), apontando o benefício da utilização de stent biliar metálico autoexpansíveis cobertos (FCSEMS) em comparação aos stents plásticos (PS). Além disso, o estudo ressalta a menor incidência de colagite, inflamação das vias biliares, após o implante de FCSEMS. Em consonância, Togawa et al. (2018) ressalta o FCSEMS como alternativa

$\mathrm{RC}: 88449$

Disponível em: https://www.nucleodoconhecimento.com.br/saude/lesoes-pancreaticas 
aos pacientes que irão ser submetidos a pancreaticoduodenectomia posteriormente, uma vez que reduz o risco de efeitos adversos pré-operatórios, como oclusão de stnet e colangite.

\section{RETARDO DO ESVAZIAMENTO GÁSTRICO APÓS PANCREATICODUODENECTOMIA}

Ao comparar os resultados das pancreaticoduodenectomias abertas (PD) e com o das laparoscópicas assistidas (LAPD), o estudo de Tian et al. (2020) apresentou em seus resultados que a LAPD não demonstra resultados inferiores perioperatórios e oncológicos de curto prazo quando comparada com o outro método. Em ambos os métodos as complicações estavam presentes, sendo o retardo do esvaziamento gástrico o principal deles. No entanto, Caruso et al. (2017) em seu estudo conclui que pacientes submetidos a LAPD apresentaram uma menor taxa de complicações, menor tempo de permanência hospitalar, relacionando a uma melhor qualidade de vida.

\section{FÍSTULA PANCREÁTICA APÓS PANCREATECTOMIA DISTAL}

O artigo de Zhang et al. (2017) mostrará que a incidência de fístula pancreática nos pacientes submetidos a pancreatomia distal laparoscópica (LDP) é 36\% (8/22) e nos submetidos a pancreatomia distal aberta (ODP) é de $25 \%$ (19/76). O resultado apresentado não demonstrou diferença significativa entre os métodos. No entanto, nenhum paciente que realizou LDP evoluiu com fístula pancreática grau $\mathrm{C}$, enquanto dois que realizaram ODP evoluíram com esta complicação. Dessa forma o estudo relaciona a utilização do grampeador endo-GIA na LDP como forma eficaz na prevenção da fístula pancreática pós-operatória, uma vez que realiza a compressão lenta do tecido pancreático.

RC: 88449

Disponível em: https://www.nucleodoconhecimento.com.br/saude/lesoes-pancreaticas 


\section{COMPLICAÇÕES GERAIS DA TERAPIA NEOADJUVANTE EM ADENOCARCINOMA PANCREÁTICO}

O estudo de Czosnyka; Borgert e Smith (2017) avaliou, por meio de uma coorte retrospectiva, as complicações gerais da terapia neoadjuvante em adenocarcinoma pancreático. Segundo o estudo, os pacientes submetidos à terapia neoadjuvante apresentaram maior necessidade de transfusão intra e pós-operatória (27,4\% vs. $20,3 \%, P<0,0001$ ), porém, associado a isto, uma menor incidência de fístulas pancreáticas. A conclusão geral foi de que a terapia aplicada gera um melhor prognóstico aos pacientes, uma vez que não ocorreu alterações na morbidade pósoperatória ou na taxa de mortalidade.

\section{COMPLICAÇÕES GERAIS DA PANCREATICODUODENECTOMIA COMBINADA COM RESSECÇÃO DA VEIA PORTA (VP) OU DA VEIA MESENTÉRICA SUPERIOR (VMS)}

O estudo de Kantor et al. (2018) conclui que a pancreaticoduodenectomia com ressecção do sistema venoso porto-mesentérico é responsável por um maior número de complicações, como aumento do tempo operatório, necessidade de transfusão sanguínea intraoperatória, sangramento, TVP e morbidade, quando comparado com a pancreaticoduodenectomia, a qual não gera estes danos. No entanto, o estudo de Fang et al. (2017) apresenta a pancreaticoduocenectomia combinada com a ressecção da veia porta (VP) ou da veia mesentérica superior (VMS) como uma melhora do prognóstico em pacientes com invasão limitada destes vasos. Defendendo ainda que, mesmo com maior tempo de cirurgia e maior perda sanguínea, esse procedimento não evidenciou maior mortalidade.

\section{CONCLUSÃO}

No presente estudo, as abordagens cirúrgicas analisadas incluíram a pancreatosplenectomia esquerda radical laparoscópica, a pancreatectomia distal

RC: 88449

Disponível em: https://www.nucleodoconhecimento.com.br/saude/lesoes-pancreaticas 
minimamente invasiva e a pancreaticoduodenectomia laparoscópica. Além disso, foram analisadas também artigos que retratavam a pancreaticoduodenectomia (PD) combinada com ressecção da veia porta (VP) ou da veia mesentérica superior (VMS), ressecção completa do tumor seguida de quimioterapia neoadjuvante, PD com excisão total do mesopâncreas e uso de stents metálicos autoexpansíveis.

Constatou-se que as principais complicações relacionadas às diferentes escolhas cirúrgicas para o tratamento de câncer de pâncreas foram o retardo do esvaziamento gástrico, a fístula do pancreática, a colangite, a necessidade de transfusão sanguínea intraoperatória, sangramento, TVP e aumento da morbidade.

De acordo com a análise realizada, a fístula pancreática está entre as principais complicações da pancreaticoduodenectomia, sendo que, ao ser realizada laparoscopicamente, encontra-se aumento das chances de fístula. Em casos de utilização de stent biliar metálico autoexpansíveis cobertos, houve menor incidência de colangite e inflamação das vias biliares. No caso das pancreaticoduodenectomias abertas e das laparoscópicas assistidas, ambas apresentaram retardo do esvaziamento gástrico como maiores complicações cirúrgicas. Ainda, pacientes submetidos à pancreatectomia distal laparoscópica (LDP) não evoluíram para fístula de grau C, o que aconteceu na pancreatectomia distal aberta (ODP). Ademais, foram encontradas também aumento do tempo operatório, necessidade de transfusão sanguínea intraoperatória, sangramento, e morbidade em pancreaticoduodenectomia com ressecção do sistema venoso porto-mesentérico e diminuição da taxa de fístula no grupo em que a terapia neoadjuvante foi feita antes da ressecção cirúrgica do adenocarcinoma pancreático.

Conclui-se, por fim, que existem diversas abordagens cirúrgicas para o câncer de pâncreas, as quais obedecem a critérios específicos, como as características morfológicas dos cistos, localização e quadro geral do paciente, não sendo nenhuma delas completamente isentas de riscos intra e pós-operatórios. O estudo em questão, portanto, além de abrir caminhos para novas discussões acerca do tema, pode auxiliar 
no estabelecimento das condutas cirúrgicas mais adequadas em quadros de câncer de pâncreas ao fazer uma análise comparativa entre a aplicação das técnicas cirúrgicas, reconhecendo as principais complicações operatórias relacionadas a lesões císticas do pâncreas, principalmente quando correlacionadas com o prognóstico do paciente.

\section{REFERÊNCIAS}

ABU HILAL, M. et al. Laparoscopic radical 'no-touch' left pancreatosplenectomy for pancreatic ductal adenocarcinoma: technique and results. Surgical endoscopy vol. 30,9 (2016): 3830-8. doi:10.1007/s00464-015-4685-9. Acesso em: 31 março 2021.

CARUSO, F. et al. Laparoscopic pancreaticoduodenectomy for tumors of the head of pancreas; 10 cases for a single center experience. European review for medical and pharmacological sciences vol. 21,17 (2017): 3745-3753. Acesso em 01 de abril de 2021.

CHEN, K. et al. Expanding laparoscopic pancreaticoduodenectomy to pancreatic-head and periampullary malignancy: major findings based on systematic review and meta-analysis. BMC Gastroenterol 18, 102 (2018). https://doi.org/10.1186/s12876-018-0830-y. Acesso em 01 de abril de 2021.

CZOSNYKA, N. M. et al. Pancreatic adenocarcinoma: effects of neoadjuvant therapy on post-pancreatectomy outcomes - an American College of Surgeons National Surgical Quality Improvement Program targeted variable review. HPB: the official journal of the International Hepato Pancreato Biliary Association vol. 19,10 (2017): 927-932. doi:10.1016/j.hpb.2017.07.001. Acesso em: 31 março 2021.

DU, F. et al. Pancreaticoduodenectomy With Arterial Approach of Total Mesenteric Resection of the Pancreas for Pancreatic Head Cancer. Gastroenterology research vol. 12,5 (2019): 256-262. doi:10.14740/gr1225. Acesso em 01 de abril de 2021.

RC: 88449

Disponível em: https://www.nucleodoconhecimento.com.br/saude/lesoes-pancreaticas 
FALQUETO, A. et al. Prevalência de lesões císticas pancreáticas em exames de imagem e associação com sinais de risco de malignidade. Radiol Bras, São Paulo, v. 51, n. 4, p. 218-224, Aug. 2018. Disponível em: $<$ http://www.scielo.br/scielo.php?script=sci_arttext\&pid=S010039842018000400218\&lng=en\&nrm=iso >. Acesso em: 25 março 2021.

FANG, J. et al. Portal vein/superior mesenteric vein resection in pancreatic cancer treatment in the elderly. Medicine vol. 96,27 (2017): e7335. doi:10.1097/MD.0000000000007335. Acesso em: 31 março 2021.

ICHIKAWA, D. et al. Laparoscopic and endoscopic co-operative surgery for nonampullary duodenal tumors. World journal of gastroenterology vol. 22,47 (2016): 10424-10431. doi:10.3748/wjg.v22.i47.10424. Acesso em: 31 março 2021.

JENSEN, E. H. et al. Pâncreas Exócrino. In: Townsend, Courtney M. Sabiston Tratado de Cirurgia- A Base Biológica da Prática Cirúrgica Moderna. 19ªed. Rio de Janeiro: Elseiver, 2015. P. 1515-1575. Acesso em: 31 março 2021.

KANTOR, O. et al. The extent of vascular resection is associated with perioperative outcome in patients undergoing pancreaticoduodenectomy. HPB: the official journal of the International Hepato Pancreato Biliary Association vol. 20,2 (2018): 140-146. doi:10.1016/j.hpb.2017.08.012. Acesso em: 31 março 2021.

LONGNECKER, D. S. Patologia das Neoplasias Pancreáticas exócrinas. UpToDate. 2021. Disponível em: https://www.uptodate.com/contents/pathology-ofexocrine-pancreatic-

neoplasms?search=neoplasias\%20pancreaticas\&source=search_result\&selectedTitl $\mathrm{e}=3 \sim 150 \&$ usage_type=default\&display_rank=3\#references. Acesso em: 25 março 2021.

MALAGELADA, J. et al. Lesões císticas pancreáticas. World Gastroenterology Organisation Global Guidelines (2019). Disponível em:

RC: 88449

Disponível em: https://www.nucleodoconhecimento.com.br/saude/lesoes-pancreaticas 
https://www.worldgastroenterology.org/UserFiles/file/guidelines/pancreatic-cysticlesions-portuguese-2019.pdf. Acesso em: 20 março 2021.

MENG, L. et al. Comparison of Laparoscopic and Open Pancreaticoduodenectomy for the Treatment of Nonpancreatic Periampullary Adenocarcinomas. Surgical laparoscopy, endoscopy \& percutaneous techniques vol. 28,1 (2018): 56-61. doi:10.1097/SLE.0000000000000504. Acesso em: 31 março 2021

REBER, H. A. Ressecção cirúrgica de lesões da cabeça do pâncreas. UpToDate. 2019. Disponível em: https://www.uptodate.com/contents/surgical-resection-oflesions-of-the-head-of-the-

pancreas?search=complica\%C3\%A7\%C3\%B5es\%20intraoperat\%C3\%B3rias\%20c \%C3\%82ncer\%20de\%20p\%C3\%A2ncreas\&source=search_result\&selectedTitle=8 150\&usage_type=default\&display_rank=8\#H176949700. Acesso em: 25 março 2021.

ROCKENBACH, B. F. Adenocarcinoma de Pâncreas. ACTA Médica, Rio Grande do Sul, v.39, n.2, p.48-53, 2018. Disponível em: https://ebooks.pucrs.br/edipucrs/acessolivre/periodicos/actamedica/assets/edicoes/2018-2/arquivos/pdf/4.pdf. Acesso em: 25 março 2021.

ROTH, S. et al. Protocol of a prospective, monocentric phase I/Il feasibility study investigating the safety of multimodality treatment with a combination of intraoperative chemotherapy and surgical resection in locally confined or borderline resectable pancreatic cancer: the combiCaRe study." BMJ open vol. 9,8 e028696. 20 Aug. 2019, doi:10.1136/bmjopen-2018-028696. Acesso em 01 de abril de 2021.

TAMURA, T. et al. Fully covered self-expandable metal stent with an antimigration system vs plastic stent for distal biliary obstruction caused by borderline resectable pancreatic cancer: A protocol for systematic review. Medicine 
vol. 99,3 (2020): e18718. doi:10.1097/MD.0000000000018718. Acesso em: 31 março 2021.

TANAKA, M. et al. Revisions of international consensus Fukuoka guidelines for the management of IPMN of the pancreas. Pancreatology. 2017 Sep-Oct;17(5):738753. doi: 10.1016/j.pan.2017.07.007. Epub 2017 Jul 13. PMID: 28735806. Acesso em: 09 abril 2021.

TIAN, F. et al. Laparoscopic assisted pancreaticoduodenectomy: an important link in the process of transition from open to total laparoscopic pancreaticoduodenectomy. BMC Surg 20, 89 (2020). https://doi.org/10.1186/s12893-020-00752-5. Acesso em 01 de abril de 2021.

TOGAWA, O. et al. Preoperative biliary drainage using a fully covered selfexpandable metallic stent for pancreatic head cancer: A prospective feasibility study. Saudi journal of gastroenterology : official journal of the Saudi Gastroenterology Association, 24(3), 151-156 (2018). https://doi.org/10.4103/sjg.SJG_448_17 . Acesso 13 de abril de 2021.

YANG, DJ. et al. The oncological safety in minimally invasive versus open distal pancreatectomy for pancreatic ductal adenocarcinoma: a systematic review and meta-analysis. Sci Rep 9, 1159 (2019). https://doi.org/10.1038/s41598-018-37617-0. Acesso em 01 de abril de 2021.

ZHANG, A. et al. Laparoscopic versus open distal pancreatectomy for pancreatic ductal adenocarcinoma: a single-center experience. Journal of Zhejiang University. Science. B vol. 18,6 (2017): 532-538. doi:10.1631/jzus.B1600541. Acesso em: 31 março 2021.

ZHANG, Q. et al. Arterial resection and reconstruction in pancreatectomy: surgical technique and outcomes. BMC Surg 19, 141 (2019). https://doi.org/10.1186/s12893-019-0560-2. Acesso em 01 de abril de 2021. 
Enviado: Maio, 2021.

Aprovado: Junho, 2021.

RC: 88449

Disponível em: https://www.nucleodoconhecimento.com.br/saude/lesoes-pancreaticas 